\title{
Toward computing gravitational initial data without elliptic solvers
}

\author{
István Rácz ${ }^{1,3}$ and Jeffrey Winicour ${ }^{2,3}$ \\ ${ }^{1}$ Wigner Research Center for Physics \\ H-1121 Budapest, Hungary \\ ${ }^{2}$ Department of Physics and Astronomy, \\ University of Pittsburg, Pittsburgh, PA, 15260, USA \\ ${ }^{3}$ Max Planck Institute for Gravitational Physics \\ Albert Einstein Institute, Golm, Germany
}

April 25, 2018

\begin{abstract}
Two new methods have been proposed for solving the gravitational constraints without using elliptic solvers by formulating them as either an algebraichyperbolic or parabolic-hyperbolic system. Here, we compare these two methods and present a unified computational infrastructure for their implementation as numerical evolution codes. An important potential application of these methods is the prescription of initial data for the simulation of black holes. This paper is meant to support progress and activity in that direction.
\end{abstract}

\section{Introduction}

Physically realistic initial data are of major importance for the numerical simulation of gravitational systems such as binary black holes. The prescription of the initial data is complicated mathematically by the nonlinear constraint equations that they must satisfy. Traditionally, the constraints have been formulated as elliptic equations, based upon the conformal treatment of the Hamiltonian constraint by Lichnerowicz [1] and later extended by York [2, 3] to treat the momentum constraint. For reviews see [4, 5].

Recently, two alternative methods for solving the constraints by means of evolution systems were introduced in [6] (see also [7, 8, 9]). In one of the proposed methods the Hamiltonian constraint is solved algebraically and the momentum constraints are expressed as a first order symmetric hyperbolic system. In the other method, the Hamiltonian constraint is formulated as a parabolic equation, with the momentum constraints again expressed as a symmetric hyperbolic system. Both of these two methods of solving the constraints have been shown to lead to well-posed problems 
for the fully nonlinear systems. Note that a well-posed problem is a necessity for a stable numerical simulation. In particular, as an important first step in establishing the viability of the algebraic-hyperbolic method, it was shown that a condition necessary for numerical stability holds globally in the case of nonlinear perturbations of Schwarzschild black hole data [10].

The details of the waveform supplied by numerical simulation of the inspiral and merger of a binary black hole is key input for interpreting the scientific content of the signals which have been observed by the LIGO-Virgo collaboration. Thus it is important that the initial data does not introduce spurious effects, e.g. the initial "junk radiation" common to all simulations based upon elliptic formulations of the constraint problem. Elliptic equations require boundary data at inner boundaries in the strong field region near the singularities inside the black holes, as well as at an outer boundary in the far field. Neglect of the tidal interaction beteween the black holes in a binary is a likely source of the junk radiation [11]. Other sources of junk radiation have been traced to the use of conformally flat iniial data. However, alternatives to conformal flatness have reduced the junk radiation content by only a factor of order 2. [11, 12]. This complicates the important problem of matching a numerical evolution to post-Netwonian parameters. Currently, this matching must be done after the junk radiation subsides.

Here we present the calculational details of two methods to solve the constraints which do not involve elliptic equations and only require data on the outer boundary, where the choice of boundary data can be guided by asymptotic flatness. In the algebraic-hyperbolic system, the 3-metric of the initial hypersurface and two components of external curvature corresponding to the radiative degrees of freedom are prescribed freely. The remaining components of external curvature are determined from the constraints. For binary black hole data, the 3-metric data can prescribed in superposed Kerr-Schild form for the individual black holes, as in [13, 14. The two components of extrinsic curvature data representing the gravitational degrees of freedom can also be prescribed by superposing the individual black hole data. In a linear theory, the superposition of such initial data for a non-radiative solution would lead to a non-radiative solution. This provides encouragement that this method might suppress junk radiation. A similar strategy is possible for the parabolic-hyperbolic system [15]. However, due to the nonlinearity of the constraints, there is no guarantee that, in the strong field region near the black holes, the constrained components of extrinsic curvature would not introduce spurious radiation. This is an issue for future work.

The constraints are solved by means of an inward "evolution" from the outer boundary by either a parabolic-hyperbolic or an algebraic-hyperbolic system of equations. Numerical stability has been demonstrated in simulations of initial data for a single perturbed black hole by means of both the algebraic-hyperbolic system [16] and the parabolic-hyperbolic system [17]. Boundary data are only necessary at the 
outer boundary, where their choice can be guided by asymptotic flatness.

Brief technical notes presenting a pseudo-code for the numerical solution of the algebraic-hyperbolic system were posted earlier [18. Since then, there has been activity in implementing both the algebraic-hyperbolic constraint system [19, 16, 20] and the parabolic-hyperbolic system [21, 17]. Because of this interest, and what we consider to be the importance of the problem, here we extend the scope of these technical notes in the following two directions. First, we present a unified treatment of the computational infrastructure necessary to implement the two approaches as numerical evolution codes. Second, we describe the details of the foliation, lapse and shift necessary for the formulation of a Cauchy problem for constructing the initial data. Our purpose is to supply the computational infrastructure for further code development and exploration.

In Sec. 2, we review the main ideas behind these two methods. In both methods, the choice of foliation of the initial Cauchy hypersurface plays an important role.

In Sec. 3, we discuss two simple choices of foliation, by spheres or by planes, for integrating the resulting constraint systems. In Sec. 4, we describe how to decompose the basic fields and their derivatives in terms of the background unit sphere geometry in the case of a spherical foliation, or a background Euclidean geometry for a planar foliation. Finally, in Sec. 5, we present the explicit form of the constraint systems in terms of spin-weighted fields defined with respect to the background geometries.

Numerical implementation of the hyperbolic equations is flexible since the evolution can proceed locally and is reversible. Parabolic equations, like elliptic equations, have strong smoothing properties and the condition for numerical stability can be relaxed by applying an implicit scheme. The precision and cost achieved in computing parabolic-hyperbolic initial data in [17] is comparable to the elliptic approach, as no iteration is necessary.

\section{Preliminaries}

In general relativity the vacuum initial data on a three-dimensional manifold $\Sigma$ consist of a Riemannian metric $h_{i j}$ and a symmetric tensor field $K_{i j}$ (the extrinsic curvature of $\Sigma)$. The pair $\left(h_{i j}, K_{i j}\right)$ is said to satisfy the vacuum constraints (see e.g. Refs. [22, 23]) if the relations

$$
\begin{array}{r}
{ }^{(3)} R+\left(K^{j}{ }_{j}\right)^{2}-K_{i j} K^{i j}=0 \\
D_{j} K^{j}{ }_{i}-D_{i} K^{j}{ }_{j}=0
\end{array}
$$

hold on $\Sigma$, where ${ }^{(3)} R$ and $D_{i}$ denote the scalar curvature and the covariant derivative operator associated with $h_{i j}$, respectively. 
The algebraic-hyperbolic and parabolic-hyperbolic constraint systems both introduce a foliation of $\Sigma$ by a one-parameter family of two-surfaces $\mathscr{S}_{\rho}$ which are the $\rho=$ const surfaces of a smooth function $\rho$ with non-vanishing gradient. The constraint system is solved by an evolution along the streamlines of a smooth vector field $\rho^{i}$ on $\Sigma$, scaled such that $\rho^{i} \partial_{i} \rho=1$. Here $\rho^{i}$ is the analogue of the time evolution vector in a Cauchy evolution.

The unit normal $\widehat{n}^{i}$ to the $\mathscr{S}_{\rho}$ level surface has the decomposition

$$
\widehat{n}^{i}=\widehat{N}^{-1}\left[\rho^{i}-\widehat{N}^{i}\right]
$$

where the "lapse" $\widehat{N}$ and "shift" $\widehat{N}^{i}$ of the evolution field $\rho^{i}$ are determined by $\widehat{n}_{i}=$ $\widehat{N} \partial_{i} \rho$ and $\widehat{N}^{i}=\widehat{\gamma}^{i}{ }_{j} \rho^{j}$. Here $\widehat{\gamma}^{i}{ }_{j}=\delta^{i}{ }_{j}-\widehat{n}^{i} \widehat{n}_{j}$ is the projection operator corresponding to the normal decomposition of the 3 -metric $h_{i j}$ into the induced metric $\widehat{\gamma}_{i j}$ of the surfaces $\mathscr{S}_{\rho}$,

$$
h_{i j}=\widehat{\gamma}_{i j}+\widehat{n}_{i} \widehat{n}_{j} .
$$

The extrinsic curvature has the analogous decomposition

$$
K_{i j}=\boldsymbol{\kappa} \widehat{n}_{i} \widehat{n}_{j}+\left[\widehat{n}_{i} \mathbf{k}_{j}+\widehat{n}_{j} \mathbf{k}_{i}\right]+\mathbf{K}_{i j}
$$

where $\boldsymbol{\kappa}=\widehat{n}^{k} \widehat{n}^{l} K_{k l}, \mathbf{k}_{i}=\widehat{\gamma}^{k}{ }_{i} \widehat{n}^{l} K_{k l}$ and $\mathbf{K}_{i j}=\widehat{\gamma}^{k}{ }_{i} \widehat{\gamma}_{j}^{l} K_{k l}$. Note that boldfaced symbols, along with the induced metric $\widehat{\gamma}_{i j}$ and the shift vector $\widehat{N}^{i}$, denote welldefined fields intrinsic to the 2 -surfaces $\mathscr{S}_{\rho}$. The reformulation of the Hamiltonian and momentum constraints (2.1) and (2.2) also involves the trace and the trace-free parts of $\mathbf{K}_{i j}$,

$$
\mathbf{K}_{l}^{l}=\widehat{\gamma}^{k l} \mathbf{K}_{k l} \quad \text { and } \quad \stackrel{\circ}{\mathbf{K}}_{i j}=\mathbf{K}_{i j}-\frac{1}{2} \widehat{\gamma}_{i j} \mathbf{K}_{l}^{l} .
$$

In addition, we denote the extrinsic curvature of $\mathscr{S}_{\rho}$ by

$$
\widehat{K}_{i j}=\widehat{\gamma}_{i}^{l} D_{l} \widehat{n}_{j}=\frac{1}{2} \mathscr{L}_{\widehat{n}} \widehat{\gamma}_{i j}=\frac{1}{2} \widehat{N}^{-1}\left[\mathscr{L}_{\rho} \hat{\gamma}_{i j}-\hat{D}_{(i} \widehat{N}_{j)}\right]
$$

The data pair $\left(h_{i j}, K_{i j}\right)$ can be replaced by the above fields $\widehat{N}, \widehat{N}^{i}, \widehat{\gamma}_{i j}, \stackrel{\circ}{\mathbf{K}}_{i j}, \boldsymbol{\kappa}, \mathbf{k}_{i}$ and $\mathbf{K}_{l}^{l}$. It is remarkable that regardless of the choice of foliation or evolution vector field the Hamiltonian and momentum constraints (2.1) and (2.2) can be re-formulated as either a parabolic-hyperbolic or algebraic-hyperbolic evolution system according to the following constructions, as formulated in [6].

\subsection{The parabolic-hyperbolic constraint system}

In the parabolic-hyperbolic approach, the Hamiltonian constraint is re-expressed as a parabolic equation (2.8) for the lapse $\widehat{N}$ of the foliation and the momentum constraint 
is recast as the first order symmetric hyperbolic system (2.9)-(2.10) for $\mathbf{k}_{i}$ and $\mathbf{K}_{l}^{l}$,

$$
\begin{aligned}
& \stackrel{\star}{K}\left[\left(\partial_{\rho} \widehat{N}\right)-\widehat{N}^{l}\left(\hat{D}_{l} \widehat{N}\right)\right]-\widehat{N}^{2}\left(\hat{D}^{l} \hat{D}_{l} \widehat{N}\right)-\mathcal{A} \widehat{N}-\mathcal{B} \widehat{N}^{3}=0, \\
& \mathscr{L}_{\hat{n}} \mathbf{k}_{i}-\frac{1}{2} \hat{D}_{i}\left(\mathbf{K}_{l}^{l}\right)-\hat{D}_{i} \boldsymbol{\kappa}+\hat{D}^{l} \stackrel{\circ}{K}_{l i}+\widehat{N}^{-1} \stackrel{\star}{K} \mathbf{k}_{i}+\left[\boldsymbol{\kappa}-\frac{1}{2}\left(\mathbf{K}_{l}^{l}\right)\right] \dot{\hat{n}}_{i}-\dot{\hat{n}}^{l} \stackrel{\circ}{K}_{l i}=0, \\
& \mathscr{L}_{\hat{n}}\left(\mathbf{K}_{l}^{l}\right)-\hat{D}^{l} \mathbf{k}_{l}-\widehat{N}^{-1} \stackrel{\star}{K}\left[\boldsymbol{\kappa}-\frac{1}{2}\left(\mathbf{K}_{l}^{l}\right)\right]+\widehat{N}^{-1} \stackrel{\circ}{K}_{k l}^{\stackrel{\star}{K}} k l+2 \dot{\hat{n}}^{l} \mathbf{k}_{l}=0
\end{aligned}
$$

Here $\hat{D}_{i}$ stands for the covariant derivative operator associated with $\hat{\gamma}_{i j}$ and $\dot{\hat{n}}_{k}=$ $\hat{n}^{l} D_{l} \hat{n}_{k}=-\hat{D}_{k}(\ln \widehat{N})$, and we introduce the notation

$$
\begin{aligned}
& \stackrel{\star}{K}_{i j}=\frac{1}{2} \mathscr{L}_{\rho} \hat{\gamma}_{i j}-\hat{D}_{(i} \widehat{N}_{j)}, \\
& \stackrel{\star}{K}=\frac{1}{2} \hat{\gamma}^{i j} \mathscr{L}_{\rho} \hat{\gamma}_{i j}-\hat{D}_{j} \widehat{N}^{j} \\
& \mathcal{A}=\left(\partial_{\rho} \stackrel{\star}{K}\right)-\widehat{N}^{l}\left(\hat{D}_{l} \stackrel{\star}{K}^{\prime}\right)+\frac{1}{2}\left[\stackrel{\star}{K}^{2}+\stackrel{\star}{K}_{k l} \stackrel{\star}{K}^{k l}\right], \\
& \mathcal{B}=-\frac{1}{2}\left[\widehat{R}+2 \boldsymbol{\kappa}\left(\mathbf{K}_{l}^{l}\right)+\frac{1}{2}\left(\mathbf{K}_{l}^{l}\right)^{2}-2 \mathbf{k}^{l} \mathbf{k}_{l}-\stackrel{\circ}{K}_{k l} \stackrel{\circ}{K}^{k l}\right] .
\end{aligned}
$$

In the form (2.8), the Hamiltonian constraint is a strongly parabolic spartial differential equation in the region of $\Sigma$ where $\stackrel{k}{K}$ is either strictly positive or strictly negative. In this case, $\rho$ plays the role of "time" and $\rho^{i}$ plays the role of a "time" evolution vector field. (For more details see [6]). Note that the sign of $\stackrel{k}{K}$ determines the stable evolution direction for the parabolic equation. It is also important that the subsystem (2.9) -(2.10) comprises a first order symmetric hyperbolic system.

As a result, the coupled parabolic-hyperbolic system (2.8)-(2.10) possesses a wellposed initial value problem for the dependent variables $\widehat{N}, \mathbf{k}_{i}, \mathbf{K}_{l}^{l}$, which guarantees the existence of a local solution. In solving (2.8)-(2.10), the variables $\widehat{N}, \mathbf{k}_{i}, \mathbf{K}_{l}^{l}$ are determined by the constraints whereas the remaining four fields $\widehat{N}^{i}, \widehat{\gamma}_{i j}, \boldsymbol{\kappa}, \stackrel{\circ}{\mathbf{K}}_{i j}$ are freely specifiable throughout $\Sigma$.

\subsection{The algebraic-hyperbolic constraint system}

An alternative approach is to recast the Hamiltonian constraint as an algebraic equation for the scalar component $\boldsymbol{\kappa}$ of $K_{i j}$. The tangential derivatives of $\boldsymbol{\kappa}$ appearing in the momentum constraint for $\mathbf{k}_{i}$ are then eliminated in terms of other variables. This results in the momentum constraint system

$$
\begin{aligned}
\mathscr{L}_{\widehat{n}}\left(\mathbf{K}_{l}^{l}\right)-\widehat{D}^{l} \mathbf{k}_{l}+2 \dot{\hat{n}}^{l} \mathbf{k}_{l}-\left[\boldsymbol{\kappa}-\frac{1}{2}\left(\mathbf{K}_{l}^{l}\right)\right]\left(\widehat{K}^{l}{ }_{l}\right)+\stackrel{\circ}{\mathbf{K}}_{k l} \widehat{K}^{k l}=0, \\
\mathscr{L}_{\widehat{n}} \mathbf{k}_{i}+\left(\mathbf{K}_{l}^{l}\right)^{-1}\left[\boldsymbol{\kappa} \widehat{D}_{i}\left(\mathbf{K}_{l}^{l}\right)-2 \mathbf{k}^{l} \widehat{D}_{i} \mathbf{k}_{l}\right]+\left(2 \mathbf{K}_{l}^{l}\right)^{-1} \widehat{D}_{i} \boldsymbol{\kappa}_{0} \\
+\left(\widehat{K}_{l}^{l}\right) \mathbf{k}_{i}+\left[\boldsymbol{\kappa}-\frac{1}{2}\left(\mathbf{K}_{l}^{l}\right)\right] \dot{\hat{n}}_{i}-\dot{\hat{n}}^{l} \stackrel{\circ}{\mathbf{K}}_{l i}+\widehat{D}^{l} \stackrel{\circ}{\mathbf{K}}_{l i}=0,
\end{aligned}
$$

whereas the Hamiltonian constraint determines $\boldsymbol{\kappa}$ algebraically by

$$
\boldsymbol{\kappa}=\left(2 \mathbf{K}_{l}^{l}\right)^{-1}\left[2 \mathbf{k}^{l} \mathbf{k}_{l}-\frac{1}{2}\left(\mathbf{K}_{l}^{l}\right)^{2}-\boldsymbol{\kappa}_{0}\right],
$$


where

$$
\boldsymbol{\kappa}_{0}={ }^{(3)} R-\stackrel{\circ}{\mathbf{K}}_{k l} \stackrel{\circ}{\mathbf{K}}^{k l} .
$$

Again, $\widehat{D}_{i}$ and $\widehat{R}$ denote the covariant derivative operator and scalar curvature associated with $\widehat{\gamma}_{i j}$, respectively, and $\dot{\widehat{n}}_{k}=\widehat{n}^{l} D_{l} \widehat{n}_{k}=-\widehat{D}_{k}(\ln \widehat{N})$. (For more details see [6]).

By virtue of (2.15)-(2.18) the four basic variables $\boldsymbol{\kappa}, \mathbf{k}_{i}, \mathbf{K}_{l}^{l}$ are determined by the constraints while the remaining eight variables, represented by the 3 -metric $h_{i j}$, consisting of $\left(\widehat{N}, \widehat{N}^{i}, \widehat{\gamma}_{i j}\right)$, and $\stackrel{\circ}{\mathbf{K}}_{i j}$, are freely specifiable throughout $\Sigma$. As a result, $\boldsymbol{\kappa}_{0}$ is determined by the freely specified variables. The constraint system (2.15)-(2.18) is symmetric hyperbolic subject to the inequality $\kappa \mathbf{K}_{l}^{l}<0$.

\section{Foliations by spheres or planes}

Two simple choices of foliations in solving the parabolic-hyperbolic system (2.8)(2.10) or the algebraic-hyperbolic system (2.15)-(2.17) are by spheres or planes, with tangential derivatives referred to a background unit sphere geometry or a background Euclidean geometry, respectively.

\subsection{Foliations by spheres and use of the $\partial$ operator}

If we chose a foliation by two-spheres it is natural to solve the constraint equations by decomposing the basic variables in terms of spin-weighted fields. In doing so, the angular derivatives are expressed in terms of the Newman-Penrose $\bar{\partial}$ and $\bar{\partial}$ operators [24, 25]. We use the notation and conventions introduced in [26, 27] throughout this paper.

The metric $q_{a b}$ on the unit sphere $\mathbb{S}^{2}$, given in standard $(\theta, \phi)$ coordinates by

$$
d s^{2}=q_{a b} \mathrm{~d} x^{a} \mathrm{~d} x^{b}=\mathrm{d} \theta^{2}+\sin ^{2} \theta \mathrm{d} \phi^{2},
$$

provides a natural background geometry. In terms of the complex stereographic coordinate

$$
z=e^{-i \phi} \cot \frac{\theta}{2}=z_{1}+\dot{\mathrm{i}} z_{2},
$$

the line element (3.1) takes the form 1

$$
d s^{2}=4(1+z \bar{z})^{-2}\left[\left(\mathrm{~d} z_{1}\right)^{2}+\left(\mathrm{d} z_{2}\right)^{2}\right] .
$$

On $\mathbb{S}^{2}$, we choose the complex dyad

$$
q^{a}=2^{-1} P\left[\left(\partial_{z_{1}}\right)^{a}+\dot{\mathrm{i}}\left(\partial_{z_{2}}\right)^{a}\right]=P\left(\partial_{\bar{z}}\right)^{a},
$$

\footnotetext{
${ }^{1}$ Only expressions for the southern hemisphere will be given explicitly. Those on the northern hemisphere can be deduced by the substitution $z_{N}=1 / z_{S}$ [26, 27].
} 
where

$$
P=1+z \bar{z}
$$

We also have

$$
q_{a}=q_{a b} q^{b}=2 P^{-1}\left[\left(\mathrm{~d} z_{1}\right)_{a}+\dot{\mathrm{i}}\left(\mathrm{d} z_{2}\right)_{a}\right]=2 P^{-1}(\mathrm{~d} z)_{a} .
$$

Note that $q^{a}$ has normalization

$$
q^{a} \bar{q}_{a}=2, \quad q^{a} q_{a}=0,
$$

and that the unit sphere metric $q_{a b}$ satisfies

$$
q_{a b}=q_{(a} \bar{q}_{b)}, \quad q^{a b}=q^{(a} \bar{q}^{b)}, \quad q^{a e} q_{e b}=\delta^{a}{ }_{b} .
$$

In these conventions, the area element on $\mathbb{S}^{2}$ is $\boldsymbol{\epsilon}_{a b}=i q_{[a} \bar{q}_{b]}$.

The Newman-Penrose $\bar{\partial}$ and $\bar{\delta}$ operators are (see e.g. (A4) in [26])

$$
\begin{aligned}
& \precsim \mathbb{L}=P^{1-s} \partial_{\bar{z}}\left(P^{s} \mathbb{L}\right) \\
& \bar{\jmath} \mathbb{L}=P^{1+s} \partial_{z}\left(P^{-s} \mathbb{L}\right),
\end{aligned}
$$

where $\mathbb{L}$ is a spin-weight $s$ field on $\mathbb{S}^{2}$ defined by

$$
\mathbb{L}=q^{a_{1}} \ldots q^{a_{s}} \mathbf{L}_{a_{1} \ldots a_{s}}
$$

for some totally symmetric traceless tensor field $\mathbf{L}_{a_{1} \ldots a_{s}}$.

As pointed out in [26, 27], this choice of $ð$ and $\bar{\partial}$ corresponds to the standard conventions in [24, 25, 27]. The action of $\check{\partial}$ and $\bar{\partial}$ on spin-weighted spherical harmonics ${ }_{s} \mathbb{Y}_{l, m}$ is given by (see e.g. (2.6)-(2.8) in [25])

$$
\begin{aligned}
\check{\partial}_{s} \mathbb{Y}_{l, m} & =\sqrt{(l-s)(l+s+1)} s+1 \\
\overline{\mathrm{\partial}}_{s} \mathbb{Y}_{l, m} & =-\sqrt{(l+s)(l-s+1)} \\
s-1 & \mathbb{Y}_{l, m}, \\
\overline{\mathrm{\partial}} \mathrm{\partial}_{s} \mathbb{Y}_{l, m} & =-(l-s)(l+s+1)_{s} \mathbb{Y}_{l, m},
\end{aligned}
$$

with

$$
{ }_{-s} \mathbb{Y}_{l, m}=(-1)^{s-m} \overline{{ }_{s} \mathbb{Y}_{l,-m}} \text {. }
$$

The $\widetilde{\partial}$ and $\bar{\varnothing}$ operators are related to the covariant derivative operator $\mathbb{D}_{a}$ associated with $q_{a b}$ by

$$
\begin{aligned}
& \check{\partial} \mathbb{L}=q^{b} q^{a_{1}} \ldots q^{a_{s}} \mathbb{D}_{b} \mathbf{L}_{\left(a_{1} \ldots a_{s}\right)}, \\
& \bar{\partial} \mathbb{L}=\bar{q}^{b} q^{a_{1}} \ldots q^{a_{s}} \mathbb{D}_{b} \mathbf{L}_{\left(a_{1} \ldots a_{s}\right)} .
\end{aligned}
$$

For a spin-weight $s$ field $\mathbb{f}$, the commutation relation for covariant derivatives on $\mathbb{S}^{2}$ implies $[\overline{\mathrm{d}}, \precsim] \mathbb{f}=2 s \mathbb{f}$. 


\subsection{Foliations by planes and the related $\partial$ and $\bar{\partial}$ operators}

In the formulation of a numerical algorithm based upon a foliation by planes it is expedient to introduce a background flat metric $q_{a b}$, analogous to the previous treatment of spheres. Accordingly, the reference two-metric $q_{a b}$ on the Euclidean plane $\mathbb{R}^{2}$ has decomposition

$$
q_{a b}=q_{(a} \bar{q}_{b)}, \quad q^{a b}=q^{(a} \bar{q}^{b)}, \quad q^{a e} q_{e b}=\delta_{b}^{a}
$$

in terms of the complex dyad

$$
q_{a}=(\mathrm{d} x)_{a}+\dot{\mathrm{i}}(\mathrm{d} y)_{a}, \quad q^{a}=\left(\partial_{x}\right)^{a}+\dot{\mathrm{i}}\left(\partial_{y}\right)^{a},
$$

with normalization

$$
q^{a} \bar{q}_{a}=2, \quad q^{a} q_{a}=0
$$

The field

$$
\mathbb{L}=q^{a_{1}} \ldots q^{a_{s}} \mathbf{L}_{a_{1} \ldots a_{s}},
$$

where $\mathbf{L}_{a_{1} \ldots a_{s}}$ is a symmetric traceless tensor field on $\mathbb{R}^{2}$, has spin-weight $s$ with respect to rotations of the dyad. Using standard complex notation, analogs of the $\check{\partial}$ and $\bar{\varnothing}$ operators can be defined in terms of the flat covariant derivative operator associated with $q_{a b}$, i.e. the partial derivative $\partial_{a}$ with respect to Cartesian coordinates $(x, y)$. This leads to the operators

$$
\begin{aligned}
& \boldsymbol{\partial} \mathbb{L}=q^{b} q^{a_{1}} \ldots q^{a_{s}} \partial_{b} \mathbf{L}_{\left(a_{1} \ldots a_{s}\right)} \\
& \overline{\boldsymbol{\partial}} \mathbb{L}=\bar{q}^{b} q^{a_{1}} \ldots q^{a_{s}} \partial_{b} \mathbf{L}_{\left(a_{1} \ldots a_{s}\right)}
\end{aligned}
$$

\subsection{The global property of the spin-weighted formalism}

Consider one of the level surfaces $\mathscr{S}_{\rho_{0}}$ of the foliation $\mathscr{S}_{\rho}$. If $\mathscr{S}_{\rho_{0}}$ is diffeomorphic to the sphere $\mathbb{S}^{2}$ we may introduce standard spherical coordinates $(\theta, \phi)$ and the unit sphere metric (3.1) on $\mathscr{S}_{\rho_{0}}$. Analogously, if $\mathscr{S}_{\rho_{0}}$ is diffeomorphic to $\mathbb{R}^{2}$, Cartesian coordinates $(x, y)$ and a Euclidean metric can be introduced. In either case, by Lie dragging these coordinates onto the leaves of the foliation $\mathscr{S}_{\rho}$ by the evolution vector field $\rho^{i}$, their values remain constant along the integral curves of $\rho^{i}$. By this process, either in terms of the coordinates $(\theta, \phi)$ or $(x, y)$, the corresponding metric $q_{a b}$ and complex dyad $q^{a}$ is defined on each of the level surfaces $\mathscr{S}_{\rho}$.

\section{The spin-weight decomposition of the basic fields}

As a consequence of the above construction, not only the coordinates $(\theta, \phi)$ or $(x, y)$ but also the complex dyad and reference metric $q_{a b}$ are Lie dragged from $\mathscr{S}_{\rho_{0}}$ onto 
the surfaces $\mathscr{S}_{\rho}$, i.e.

$$
\mathscr{L}_{\rho} q^{a}=0, \quad \mathscr{L}_{\rho} q_{a}=0 \quad \text { and } \quad \mathscr{L}_{\rho} q_{a b}=0 .
$$

It is a convenient consequence of this construction that there is a single common treatment of the two cases based on foliations by spheres or planes. Either case can be derived from the other by the simple replacements $(\theta, \phi) \longleftrightarrow(x, y)$ and $\partial \longleftrightarrow \boldsymbol{\partial}$. Accordingly, the calculations below will be presented exclusively for the case of a spherical foliation.

\subsection{The decomposition of the metric $\widehat{\gamma}_{a b}$}

The metric $\widehat{\gamma}_{a b}$ induced on the $\mathscr{S}_{\rho}$ level surfaces can be decomposed as

$$
\widehat{\gamma}_{a b}=\mathrm{a} q_{a b}+\stackrel{\circ}{\gamma}_{a b}
$$

where

$$
\mathrm{a}=\frac{1}{2} \widehat{\gamma}_{a b} q^{a} \bar{q}^{b}
$$

is a positive, spin-weight zero function on $\mathscr{S}_{\rho}$ and $\stackrel{\circ}{\gamma}_{a b}$ is its trace-free part, i.e.

$$
\stackrel{\circ}{\gamma}_{a b}=\left[\delta_{a}^{e} \delta_{b}^{f}-\frac{1}{2} q_{a b} q^{e f}\right] \widehat{\gamma}_{e f}=\widehat{\gamma}_{a b}-\mathrm{a} q_{a b} .
$$

Since $\stackrel{\circ}{\gamma}_{a b}$ is symmetric and trace-free it has the decomposition

$$
\stackrel{\circ}{\gamma}_{a b}=\frac{1}{2}\left[\varpi \bar{q}_{a} \bar{q}_{b}+\bar{\varpi} q_{a} q_{b}\right]
$$

in terms of the spin-weight 2 function

$$
\mathrm{b}=\frac{1}{2} \widehat{\gamma}_{a b} q^{a} q^{b}=\frac{1}{2} \stackrel{\circ}{\gamma}_{a b} q^{a} q^{b} .
$$

The inverse metric has the decomposition

$$
\widehat{\gamma}^{a b}=\mathbb{d}^{-1}\left\{\mathrm{a} q^{a b}-\frac{1}{2}\left[\mathrm{~b} \bar{q}^{a} \bar{q}^{b}+\overline{\mathrm{b}} q^{a} q^{b}\right]\right\},
$$

where

$$
\mathbb{d}=\mathrm{a}^{2}-\mathbb{b} \overline{\mathrm{b}}
$$

is the ratio $\operatorname{det}\left(\widehat{\gamma}_{a b}\right) / \operatorname{det}\left(q_{a b}\right)$ of the determinants of $\widehat{\gamma}_{a b}$ and $q_{a b}$.

As an immediate application, using (3.8), (4.2) and (4.4), along with the notation

$$
\mathbb{k}=q^{l} \mathbf{k}_{l}, \quad \overline{\mathbb{k}}=\bar{q}^{l} \mathbf{k}_{l},
$$

$\mathbf{k}^{l} \mathbf{k}_{l}$ can be expressed as

$$
\begin{aligned}
\mathbf{k}^{l} \mathbf{k}_{l} & =\widehat{\gamma}^{k l} \mathbf{k}_{k} \mathbf{k}_{l}=\frac{1}{2} \mathbb{d}^{-1}\left\{\mathrm{a}\left(q^{k} \bar{q}^{l}+q^{l} \bar{q}^{k}\right)-\left[\bar{b} \bar{q}^{k} \bar{q}^{l}+\overline{\mathrm{b}} q^{l} q^{k}\right]\right\} \mathbf{k}_{k} \mathbf{k}_{l} \\
& =\frac{1}{2} \mathbb{d}^{-1}\left[2 \mathrm{a} \mathbb{k} \overline{\mathbb{k}}-\mathbb{b}_{\mathbb{k}^{2}}-\overline{\mathrm{b}} \mathbb{k}^{2}\right] .
\end{aligned}
$$




\subsection{Terms involving the covariant derivative $\widehat{D}_{a}$}

The covariant derivative operators $\widehat{D}_{a}$ and $\mathbb{D}_{a}$ can be related by the tensor field

$$
C^{e}{ }_{a b}=\frac{1}{2} \widehat{\gamma}^{e f}\left\{\mathbb{D}_{a} \widehat{\gamma}_{f b}+\mathbb{D}_{b} \widehat{\gamma}_{a f}-\mathbb{D}_{f} \widehat{\gamma}_{a b}\right\}
$$

(See e.g. (3.1.28) and (D.3) in [23].) In particular,

$$
\widehat{D}_{a} \mathbf{k}_{b}=\mathbb{D}_{a} \mathbf{k}_{b}-C_{a b}^{e} \mathbf{k}_{e}
$$

and thereby

$$
\begin{aligned}
\widehat{D}^{l} \mathbf{k}_{l} & =\widehat{\gamma}^{k l} \widehat{D}_{k} \mathbf{k}_{l}=\frac{1}{2} \mathbb{d}^{-1}\left\{\mathrm{a}\left(q^{k} \bar{q}^{l}+q^{l} \bar{q}^{k}\right)-\left[\mathfrak{b} \bar{q}^{k} \bar{q}^{l}+\overline{\mathrm{b}} q^{l} q^{k}\right]\right\} \widehat{D}_{k} \mathbf{k}_{l} \\
& =\frac{1}{4} \mathbb{d}^{-1}\left\{2 \mathrm{a}(\overline{\mathrm{d}} \overline{\mathbb{k}}-\mathbb{B} \overline{\mathbb{k}})-\mathbb{b}(2 \bar{\partial} \overline{\mathbb{k}}-\overline{\mathbb{C}} \mathbb{k}-\overline{\mathbb{A}} \overline{\mathbb{k}})+{ }^{\prime \prime} C C "\right\},
\end{aligned}
$$

where

$$
\begin{aligned}
& \mathbb{A}=q^{a} q^{b} C^{e}{ }_{a b} \bar{q}_{e}=\mathbb{d}^{-1}\{\mathrm{a}[2 \partial \mathrm{d}-\overline{\widetilde{\partial}} \mathrm{b}]-\overline{\mathrm{b}} \partial \mathrm{b}\} \\
& \mathbb{B}=\bar{q}^{a} q^{b} C^{e}{ }_{a b} q_{e}=\mathbb{d}^{-1}\{\mathrm{a} \bar{\partial} \mathrm{b}-\mathfrak{b} \partial \overline{\mathrm{b}}\} \\
& \mathbb{C}=q^{a} q^{b} C^{e}{ }_{a b} q_{e}=\mathbb{d}^{-1}\{\mathrm{a} \partial \mathfrak{b}-\mathfrak{b}[2 \partial \mathrm{a}-\bar{\partial} \mathfrak{b}]\} .
\end{aligned}
$$

Hereafter " $C C$ " stands for the complex conjugate of the previous terms within the same brackets or parentheses.

We also obtain the relation

$$
\begin{aligned}
& {\left[2 \mathbf{k}^{l} \widehat{D}_{i} \mathbf{k}_{l}\right] q^{i} }=\left[2 \widehat{\gamma}^{k l} \mathbf{k}_{k} \widehat{D}_{i} \mathbf{k}_{l}\right] q^{i} \\
&= {\left[\mathbb{d}^{-1}\left\{\mathrm{a}\left(q^{k} \bar{q}^{l}+q^{l} \bar{q}^{k}\right)-\left[\bar{b} \bar{q}^{k} \bar{q}^{l}+\overline{\mathrm{b}} q^{l} q^{k}\right]\right\} \mathbf{k}_{k} \widehat{D}_{i} \mathbf{k}_{l}\right] q^{i} } \\
&=\frac{1}{2} \mathbb{d}^{-1}\{(\mathrm{a} \mathbb{k}-\mathbb{b} \overline{\mathbb{k}})[2 ð \overline{\mathbb{k}}-\overline{\mathbb{B}} \mathbb{k}-\mathbb{B} \overline{\mathbb{k}}]+(\mathrm{a} \overline{\mathbb{k}}-\overline{\mathrm{b}} \mathbb{k})[2 ð \mathbb{k}-\mathbb{C} \overline{\mathbb{k}}-\mathbb{A} \mathbb{k}]\} .
\end{aligned}
$$

\subsection{The scalar curvature ${ }^{(3)} R$}

In expressing the scalar curvature ${ }^{(3)} R$ in terms of spin-weighted fields we use the relation

$$
{ }^{(3)} R=\widehat{R}-\left[2 \mathscr{L}_{\widehat{n}}\left(\widehat{K}_{l}^{l}\right)+\left(\widehat{K}_{l}^{l}\right)^{2}+\widehat{K}_{k l} \widehat{K}^{k l}+2 \widehat{N}^{-1} \widehat{D}^{l} \widehat{D}_{l} \widehat{N}\right]
$$

where $\widehat{R}$ is the scalar curvature of the metric $\widehat{\gamma}_{a b}$, given by

$$
\widehat{R}=\widehat{\mathbb{R}}=\frac{1}{2} \mathrm{a}^{-1}\left(\mathbb{R}-\left\{\partial \overline{\mathbb{B}}-\bar{\partial} \mathbb{A}-\frac{1}{2}[\mathbb{C} \overline{\mathbb{C}}-\mathbb{B} \overline{\mathbb{B}}]\right\}\right)
$$

in terms of the scalar curvature $\mathbb{R}$ associated with $\mathbb{D}_{a}$. (For the foliation by spheres $\mathbb{R}=2$, and for planes $\mathbb{R}=0$.) The basic field variables used in recasting the constraint equations are collected in Table1. 


\begin{tabular}{|c|c|c|}
\hline notation & definition & spin-weight \\
\hline $\mathrm{a}$ & $\frac{1}{2} q^{i} \bar{q}^{j} \widehat{\gamma}_{i j}$ & 0 \\
\hline $\mathrm{b}$ & $\frac{1}{2} q^{i} q^{j} \widehat{\gamma}_{i j}$ & 2 \\
\hline $\mathbb{d}$ & $\mathrm{a}^{2}-\mathrm{b} \overline{\mathrm{b}}$ & 0 \\
\hline $\mathbb{k}$ & $q^{i} \mathbf{k}_{i}$ & 1 \\
\hline $\mathbb{A}$ & $q^{a} q^{b} C_{a b}^{e} \bar{q}_{e}=\mathbb{d}^{-1}\left\{\mathrm{a}\left[2 \partial_{\mathrm{a} a}-\overline{\mathrm{d}} \mathrm{b}\right]-\overline{\mathrm{b}} \partial \mathrm{b}\right\}$ & 1 \\
\hline $\mathbb{B}$ & $\bar{q}^{a} q^{b} C_{a b}^{e} q_{e}=\mathbb{d}^{-1}\{a \bar{a} \bar{\partial}-\mathfrak{b} \partial \bar{b}\}$ & 1 \\
\hline $\mathbb{C}$ & $q^{a} q^{b} C_{a b}^{e} q_{e}=\mathbb{d}^{-1}\{\mathrm{a} \partial \mathfrak{b}-\mathfrak{b}[2 \partial \mathrm{a}-\bar{\partial} \mathrm{b}]\}$ & 3 \\
\hline$\widehat{\mathbb{R}}$ & $\frac{1}{2} \mathrm{a}^{-1}\left(\mathbb{R}-\left\{\partial \overline{\mathbb{B}}-\bar{\circlearrowright} \mathbb{A}-\frac{1}{2}[\mathbb{C} \overline{\mathbb{C}}-\mathbb{B} \overline{\mathbb{B}}]\right\}\right)$ & 0 \\
\hline$\widehat{\mathbb{N}}$ & $\widehat{N}$ & 0 \\
\hline $\mathbb{N}$ & $q^{i} \widehat{N}_{i}=q^{i} \widehat{\gamma}_{i j} \widehat{N}^{j}$ & 1 \\
\hline$\widetilde{\mathbb{N}}$ & $q_{i} \widehat{N}^{i}=q_{i} \widehat{\gamma}^{i j} \widehat{N}_{j}=\mathbb{d}^{-1}(\mathrm{a} \mathbb{N}-\mathfrak{b} \overline{\mathbb{N}})$ & 1 \\
\hline $\mathbb{K}$ & $\widehat{\gamma}^{k l} \mathbf{K}_{k l}$ & 0 \\
\hline$\stackrel{\circ}{\mathbb{K}}_{q q}$ & $q^{k} q^{l} \stackrel{\circ}{\mathbf{K}}_{k l}$ & 2 \\
\hline$\stackrel{\circ}{\mathbb{K}}_{q \bar{q}}$ & $q^{k} \bar{q}^{l} \stackrel{\circ}{\mathbf{K}}_{k l}=(2 \mathrm{a})^{-1}\left[\bar{b} \overline{\stackrel{\circ}{K}}_{q q}+\overline{\mathbb{b}} \stackrel{\circ}{\mathbb{K}}_{q q}\right]$ & 0 \\
\hline$\widehat{\mathbb{K}}$ & $\widehat{K}_{l}^{l}=\widehat{\gamma}^{i j} \widehat{K}_{i j}$ & 0 \\
\hline$\widehat{\mathbb{K}}_{q q}$ & $q^{i} q^{j} \widehat{K}_{i j}=\frac{1}{2} \widehat{\mathbb{N}}^{-1}\left\{2 \partial_{\rho} \mathfrak{b}-2 \partial \mathbb{N}+\mathbb{C} \overline{\mathbb{N}}+\mathbb{A} \mathbb{N}\right\}$ & 2 \\
\hline$\widehat{\mathbb{K}}_{q \bar{q}}$ & $q^{k} \bar{q}^{l} \widehat{K}_{k l}=\mathrm{a}^{-1}\left\{\mathbb{d} \cdot \widehat{\mathbb{K}}+\frac{1}{2}\left[\mathfrak{b} \widehat{\mathbb{K}}_{q q}+\overline{\mathrm{b}} \widehat{\mathbb{K}}_{q q}\right]\right\}$ & 0 \\
\hline$\stackrel{\star}{\mathbb{K}}$ & $\stackrel{\star}{K}_{l}^{l}=\widehat{\gamma}^{i j} \stackrel{\star}{K}_{i j}$ & 0 \\
\hline$\stackrel{\star}{\mathbb{K}}_{q q}$ & $q^{i} q^{j} \stackrel{\star}{K}_{i j}=\frac{1}{2}\left\{2 \partial_{\rho} \mathfrak{b}-2 \partial \mathbb{N}+\mathbb{C} \overline{\mathbb{N}}+\mathbb{A} \mathbb{N}\right\}$ & 2 \\
\hline$\stackrel{\star \star}{\mathbb{K}}_{q \bar{q}}$ & 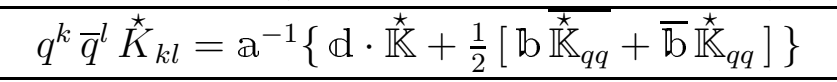 & 0 \\
\hline
\end{tabular}

Table 1: The new variables used in recasting the constraints. For detailed derivations of these and other complicated expressions see the Appendix. 


\section{The constraints in terms of the new variables}

We now present the explicit form of the constraints in terms of the spin-weighted fields and their derivatives introduced in the previous sections.

\subsection{The parabolic-hyperbolic system}

Application of the spin-weight decomposition of the basic variables leads to the following form of the parabolic-hyperbolic system (2.8)-(2.10),

$$
\begin{aligned}
& \stackrel{\star}{\mathbb{K}}\left[\partial_{\rho} \widehat{\mathbb{N}}-\frac{1}{2} \widetilde{\mathbb{N}}(\bar{\partial} \widehat{\mathbb{N}})-\frac{1}{2} \overline{\widetilde{N}}(ð \widehat{\mathbb{N}})\right] \\
& -\frac{1}{2} \mathbb{d}^{-1} \widehat{\mathbb{N}}^{2}\left[a\{(ð \bar{\partial} \widehat{\mathbb{N}})-\mathbb{B}(\bar{\partial} \widehat{\mathbb{N}})\}-\rrbracket\left\{\left(\bar{\partial}^{2} \widehat{\mathbb{N}}\right)-\frac{1}{2} \overline{\mathbb{A}}(\bar{\partial} \widehat{\mathbb{N}})-\frac{1}{2} \overline{\mathbb{C}}(ð \widehat{\mathbb{N}})\right\}+" C C "\right] \\
& -\mathcal{A} \widehat{\mathbb{N}}-\mathcal{B} \widehat{\mathbb{N}}^{3}=0 \text {, } \\
& \partial_{\rho} \mathbb{k}-\frac{1}{2} \widetilde{\mathbb{N}}(\bar{\partial} \mathbb{k})-\frac{1}{2} \widetilde{\widetilde{N}}(ð \mathbb{k})-\frac{1}{2} \widehat{\mathbb{N}} \partial \mathbb{K}+\mathbb{f}_{\mathbb{k}}=0,
\end{aligned}
$$

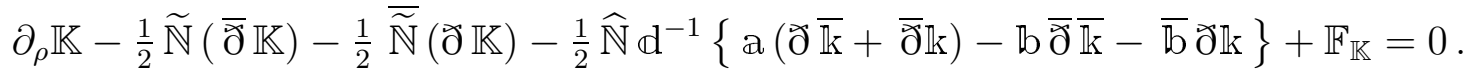

In (5.2) -(5.3), the lower order source terms $\mathbb{f}_{\mathbb{k}}$ and $\mathbb{F}_{\widehat{\mathbb{K}}}$ have spin-weight 1 and 0 , respectively, and the coefficients $\mathcal{A}$ and $\mathcal{B}$ have spin-weight 0 , on each surface $\mathscr{S}_{\rho}$. They are smooth functions of the constrained variables $\widehat{\mathbb{N}}, \mathbb{K}, \mathbb{k}$ and the freely specified variables $a, \mathfrak{b}, \mathbb{N}, \boldsymbol{\kappa}, \stackrel{\circ}{K}_{q q}$, along with the $\precsim$, $\bar{\varnothing}$ and $\rho$-derivatives of the free variables. Their explicit forms are

$$
\begin{aligned}
& \mathbb{f}_{\mathbb{k}}=-\frac{1}{2}[\mathbb{k} \partial \overline{\widetilde{\mathbb{N}}}+\overline{\mathbb{k}} \partial \widetilde{\mathbb{N}}]-\left[\boldsymbol{\kappa}-\frac{1}{2} \mathbb{K}\right] \partial \widehat{\mathbb{N}}+\mathbb{p} \\
& +\widehat{\mathbb{N}}\left[-ð \boldsymbol{\kappa}+\widehat{\mathbb{N}}^{-1} \stackrel{\star}{\mathbb{K}} \mathbb{k}-q^{i} \dot{\hat{n}}^{l} \stackrel{\circ}{\mathbf{K}}_{l i}+q^{i} \widehat{D}^{l} \stackrel{\circ}{\mathbf{K}}_{l i}\right], \\
& \mathbb{F}_{\mathbb{K}}=\frac{1}{4} \widehat{\mathbb{N}} \mathbb{d}^{-1}\left\{2 \mathrm{a} \mathbb{B} \overline{\mathbb{k}}-\mathbb{b}(\overline{\mathbb{C}} \mathbb{k}+\overline{\mathbb{A}} \overline{\mathbb{k}})-\left\{\mathbb{p}_{\rho}-\frac{1}{2}[\widetilde{\mathbb{N}} \overline{\mathbb{P}}+\overline{\widetilde{\mathbb{N}}} \mathbb{p}]\right\}+" C C "\right\} \\
& -\mathbb{d}^{-1}[(\mathrm{a} \overline{\mathbb{K}}-\overline{\mathrm{b}} \mathbb{k}) \partial \widehat{\mathbb{N}}+“ C C "]+\left[\stackrel{\circ}{\mathbf{K}}_{i j} \stackrel{\star}{K^{i j}}-\left(\boldsymbol{\kappa}-\frac{1}{2} \mathbb{K}\right) \stackrel{\star}{\mathbb{K}}\right],
\end{aligned}
$$

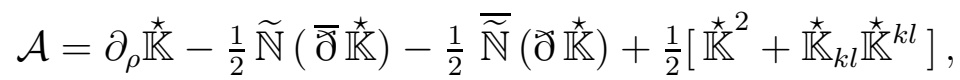

$$
\begin{aligned}
& \mathcal{B}=-\frac{1}{2}\left[\widehat{\mathbb{R}}+2 \kappa \mathbb{K}+\frac{1}{2} \mathbb{K}^{2}-\mathbb{d}^{-1}\left[2 \text { a } \mathbb{k} \overline{\mathbb{k}}-b^{2} \overline{\mathbb{k}}^{2}-\overline{\mathfrak{b}} \mathbb{k}^{2}\right]-\stackrel{\circ}{\mathbf{K}}_{k l} \stackrel{\circ}{\mathbf{K}}^{k l}\right] \text {, }
\end{aligned}
$$


where the explicit forms of the new terms introduced in (5.4)-(5.7) are

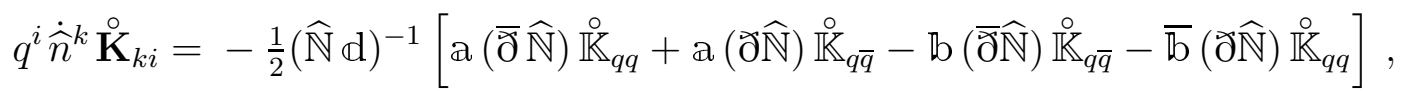

$$
\begin{aligned}
& q^{i} \widehat{D}^{k} \stackrel{\circ}{\mathbf{K}}_{k i}=\frac{1}{2 \mathbb{d}}\left(\mathrm{a} \bar{\partial} \stackrel{\circ}{\mathbb{K}}_{q q}+\mathrm{a} \partial \stackrel{\circ}{\mathbb{K}}_{q \bar{q}}-\mathrm{b} \bar{\partial} \stackrel{\circ}{\mathbb{K}}_{q \bar{q}}-\overline{\mathrm{b}} \partial \stackrel{\circ}{\mathbb{K}_{q q}}\right)+\frac{\overline{\mathrm{b}}}{2 \mathbb{d}}\left(\mathbb{A} \stackrel{\circ}{\mathbb{K}}_{q q}+\mathbb{C} \stackrel{\circ}{\mathbb{K}}_{q \bar{q}}\right) \\
& -\frac{\mathrm{a}}{4 \mathbb{d}}\left(3 \overline{\mathbb{B}} \stackrel{\circ}{\mathbb{K}}_{q q}+3 \mathbb{B} \stackrel{\circ}{\mathbb{K}}_{q \bar{q}}+\mathbb{A} \stackrel{\circ}{\mathbb{K}}_{q \bar{q}}+\mathbb{C} \overline{\mathbb{R}^{\mathbb{K}}}\right) \\
& +\frac{\mathfrak{b}}{4 \mathbb{d}}\left(\overline{\mathbb{C}} \stackrel{\circ}{\mathbb{K}}_{q q}+\overline{\mathbb{A}} \stackrel{\circ}{\mathbb{K}}_{q \bar{q}}+\overline{\mathbb{B}} \stackrel{\circ}{\mathbb{K}}_{q \bar{q}}+\mathbb{B} \overline{\stackrel{\circ}{\mathbb{K}}}_{q q}\right), \\
& \stackrel{\circ}{\mathbf{K}}_{i j} \stackrel{\star}{K}^{i j}=\frac{1}{4} \mathbb{d}^{-2}\left\{2 \stackrel{\circ}{\mathbb{K}}_{q \bar{q}}\left[\left(\mathrm{a}^{2}+\mathrm{b} \overline{\mathrm{b}}\right) \stackrel{\star}{\mathbb{K}}_{q \bar{q}}-\mathrm{a}\left(\overline{\mathrm{b}} \stackrel{\star}{\mathbb{K}}_{q q}+\mathrm{b} \overline{\stackrel{\star}{\mathbb{K}}}_{q q}\right)\right]\right. \\
& \left.+\left[\overline{\mathbb{K}_{q q}}\left(\mathrm{a}^{2} \stackrel{\star}{\mathbb{K}}_{q q}+\mathrm{b}^{2} \overline{\mathbb{K}_{q q}}-2 \mathrm{a} \mathrm{b} \stackrel{\star}{\mathbb{K}}_{q \bar{q}}\right)+" C C "\right]\right\}, \\
& \stackrel{\star}{K}_{i j} \stackrel{\star}{K}^{i j}=\frac{1}{4} \mathbb{d}^{-2}\left\{\left[\overline{\overleftarrow{\mathbb{K}}_{q q}}\left(\mathrm{a}^{2} \stackrel{\star}{\mathbb{K}_{q q}}+\mathrm{b}^{2} \overline{\overline{\mathbb{K}}_{q q}}-4 \mathrm{a} \mathfrak{b} \stackrel{\star}{\mathbb{K}_{q \bar{q}}}\right)+{ }^{*} C C \text { " }\right]\right. \\
& \left.+2\left(\mathrm{a}^{2}+\mathbb{b} \overline{\mathrm{b}}\right) \stackrel{\star}{\mathbb{K}_{q \bar{q}}^{2}}\right\},
\end{aligned}
$$

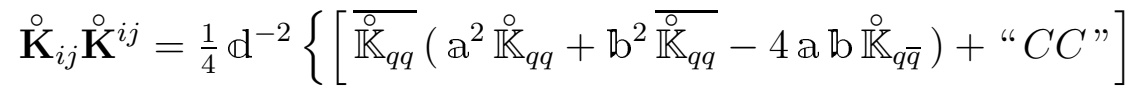

$$
\begin{aligned}
& \left.+2\left(\mathrm{a}^{2}+\sqrt[b]{\bar{b}}\right) \stackrel{\circ}{\mathbb{K}}_{q \bar{q}}^{2}\right\} \text {. }
\end{aligned}
$$

For detailed derivation of these relations see the Appendix.

\subsection{The algebraic-hyperbolic system}

Application of the spin-weight decomposition of the basic variables introduced in Table1 leads to the following form of the algebraic-hyperbolic constraints,

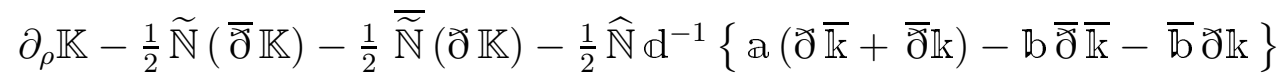

$$
\begin{aligned}
& +\mathbb{F}_{\mathbb{K}}=0, \\
& \partial_{\rho} \mathbb{k}-\frac{1}{2} \widetilde{\mathbb{N}}(\bar{\partial} \mathbb{k})-\frac{1}{2} \overline{\widetilde{N}}(ð \mathbb{k})+\widehat{\mathbb{N}}(\mathbb{K})^{-1}\left\{\kappa(ð \mathbb{K})-\mathbb{d}^{-1}[(\mathrm{a} \mathbb{k}-\mathbb{b} \overline{\mathbb{k}})(ð \overline{\mathbb{k}})\right. \\
& +(\mathrm{a} \overline{\mathbb{k}}-\overline{\mathrm{b}} \mathbb{k})(\check{\partial} \mathfrak{k})]\}+\mathbb{f}_{\mathbb{k}}=0, \\
& \boldsymbol{\kappa}=(2 \mathbb{K})^{-1}\left[\mathbb{d}^{-1}\left(2 \mathrm{a} \mathbb{k} \overline{\mathbb{k}}-\mathfrak{b} \overline{\mathbb{k}}^{2}-\overline{\mathfrak{b}} \mathbb{k}^{2}\right)-\frac{1}{2} \mathbb{K}^{2}-\boldsymbol{\kappa}_{0}\right] \text {, }
\end{aligned}
$$

where, in virtue of (2.18), $\boldsymbol{\kappa}_{0}$ can be evaluated by applying (4.16), (4.17), (A.14), (A.25), (A.34) and (A.36).

In (5.13) - (5.14), the lower order source terms $\mathbb{F}_{\mathbb{K}}$ and $\mathbb{f}_{\mathbb{k}}$ have spin-weight 0 and 1 , respectively. They are both smooth undifferentiated functions of the constrained 
variables $\boldsymbol{\kappa}, \mathbb{K}, \mathbb{k}$; and they are also smooth functions of the freely specifiable variables $\mathrm{a}, \mathfrak{b}, \widehat{\mathbb{N}}, \mathbb{N}, \mathbb{K}_{q q}$ and their derivatives. The explicit forms of the forcing terms are

$$
\begin{aligned}
& \mathbb{F}_{\mathbb{K}}=\frac{1}{4} \widehat{\mathbb{N}} \mathbb{d}^{-1}\{2 \mathrm{a} \mathbb{B} \overline{\mathbb{k}}-\mathfrak{b}(\overline{\mathbb{C}} \mathbb{k}+\overline{\mathbb{A}} \overline{\mathbb{k}})+" C C "\} \\
& -\mathbb{d}^{-1}[(\mathrm{a} \overline{\mathbb{k}}-\overline{\mathrm{b}} \mathbb{k}) \partial \widehat{\mathbb{N}}+“ C C "]+\widehat{\mathbb{N}}\left[\stackrel{\circ}{\mathbf{K}}_{i j} \widehat{K}^{i j}-\left(\boldsymbol{\kappa}-\frac{1}{2} \mathbb{K}\right) \widehat{\mathbb{K}}\right] \text {, } \\
& \mathbb{f}_{\mathbb{k}}=-\frac{1}{2}\left[\mathbb{k}_{k} \partial \overline{\widetilde{\mathbb{N}}}+\overline{\mathbb{k}} \partial \widetilde{\mathbb{N}}\right] \\
& +\frac{1}{2} \widehat{\mathbb{N}}(\mathbb{d} \mathbb{K})^{-1}[(a \mathbb{k}-\mathbb{b} \overline{\mathbb{k}})(\overline{\mathbb{B}} \mathbb{k}+\mathbb{B} \overline{\mathbb{k}})+(a \overline{\mathbb{k}}-\overline{\mathrm{b}} \mathbb{k})(\mathbb{C} \overline{\mathbb{k}}+\mathbb{A} \mathbb{k})] \text {, } \\
& -\left[\boldsymbol{\kappa}-\frac{1}{2} \mathbb{K}\right] ð \widehat{\mathbb{N}}+\widehat{\mathbb{N}}\left[\frac{1}{2} \mathbb{K}^{-1} \partial \boldsymbol{\kappa}_{0}+\widehat{\mathbb{K}} \mathbb{k}-q^{i} \dot{\widehat{n}}^{l} \stackrel{\circ}{\mathbf{K}}_{l i}+q^{i} \widehat{D}^{l} \stackrel{\circ}{\mathbf{K}}_{l i}\right] \text {, }
\end{aligned}
$$

where, , in virtue of (A.34)

$$
\begin{aligned}
\stackrel{\circ}{\mathbf{K}}_{i j} \widehat{K}^{i j}=\frac{1}{4} \mathbb{d}^{-2}[ & \left.2 \stackrel{\circ}{\mathbb{K}}_{q \bar{q}}\left(\left[\mathrm{a}^{2}+\mathrm{b} \overline{\mathrm{b}}\right)\right] \widehat{\mathbb{K}}_{q \bar{q}}-\mathrm{a}\left[\overline{\mathrm{b}} \widehat{\mathbb{K}}_{q q}+\mathbb{b} \overline{\widehat{\mathbb{K}}_{q q}}\right]\right) \\
& \left.+\left\{\overline{\mathbb{K}_{q q}}\left[\mathrm{a}^{2} \widehat{\mathbb{K}}_{q q}+b^{2} \widehat{\mathbb{K}}_{q q}-2 \mathrm{a} \mathrm{b} \widehat{\mathbb{K}}_{q \bar{q}}\right]+" C C "\right\}\right]
\end{aligned}
$$

whereas, $q^{i} \dot{\hat{n}}^{l} \stackrel{\circ}{K}_{l i}$ and $q^{i} \widehat{D}^{l} \stackrel{\circ}{\mathbf{K}}_{l i}$ have been explicitly given in (5.8)-(5.9) $)$.

\section{$6 \quad$ Future prospects}

We have presented the computational infrastructure necessary for a numerical code to solve the algebraic-hyperbolic or parabolic-hyperbolic versions of the constraint equations. The derivatives tangential to the foliation can be approximated by a finite difference or pseudo-spectral representation of the $\partial$ or $\boldsymbol{\partial}$ operators. The "radial" integrations along the $\rho$-streamlines can then approximated on a finite grid by a coupled system of ordinary differential equations by applying the method of lines. Although the analytic theory shows that the constraint systems are well-posed under appropriate conditions, the issue of a global solution to a nonlinear problem is normally best explored by numerical techniques. (A relevant exception is the possibility to apply energy methods to prove global existence of solutions to both evolutionary versions of the constraints for initial data near Schwarzschild [28]).

The numerical investigations carried out so far provide some promise that these evolutionary methods are viable alternatives to the elliptic approach for the construction of initial data. In an investigation of whether the algebraic-hyperbolic system is consistent with asymptotic flatness, a code developed in [19] using a spectral representation of the spin-weighted harmonics [29] has simulated stable evolutions in the outward $\rho$ direction for nonlinear perturbations of Schwarzschild data. Work in progress in [16, 20] using finite difference codes has shown that the inward evolution of nonlinear Schwarzschild perturbations can be stably extended to the interior of 
the horizon. The parabolic-hyperbolic method, combined with a foliation by spherical surfaces, has been successfully applied in computing nonlinear perturbations of Minkowski initial data and using that data to carry out a corresponding constrained time evolution [21]. For the single black hole case, it has been demonstrated in [17] that the parabolic-hyperbolic system can be stably and accurately integrated numerically. A detailed investigation of generic single but boosted and spinning black holes verified that the full parameter space could be effectively explored without the use of boundary conditions in the strong field regime.

The ultimate utility of this new approach would be its extension to multiple black holes. A major concern in such a scheme is the effect of caustics, where the ingoing $\rho$ streamlines focus, or a cross-over surface $\mathscr{S}_{X}$ where those streamlines from opposing points of the outer boundary meet. For a single black hole, the $\rho$ streamlines can be chosen so that any caustics and crossovers are inside the apparent horizon, where the interior can be excised. The excision of some interior singularity seems to be a necessity for the application to a spherical foliation [31.

Formally, these methods can be applied to the multiple black hole problem using for the freely specified variables, say, a modification of the superimposed Kerr-Schild data proposed in [13, 14, 10, 30, 15]. Among other things, the success of a numerical implementation would depend upon a judicious choice of the foliation $\mathscr{S}_{\rho}$ and the $\rho$-streamlines along which the evolution proceeds. This is akin to choosing the lapse and shift for a timelike Cauchy evolution. For binary black hole data, although the caustics can be arranged to lie inside the black holes, the crossover surface will in general span the region between them. In that case, unless $\mathscr{S}_{X}$ can be chosen to be a surface of reflection symmetry, as in the case of data for binary black holes with parallel or anti-parallel spins the inward evolution can produce a discontinuity on $\mathscr{S}_{X}$. It is anticipated that the methods developed in [30] will be helpful in computing initial data for binary systems with generic spins and velocities.

Considerable numerical experimentation will be necessary to deal with the technical issues. The understanding of the analytic properties and numerical implementation of the elliptic formulation of the constrains has had a long and complicated history. Unlike the iterative global nature of elliptic solvers, the flexibility of hyperbolic systems to proceed locally may be of advantage here. Since hyperbolic evolution of the constraint system can also proceed in the outward $\rho$-direction, discontinuities on the crossover surface $\mathscr{S}_{X}$ can possibly be smoothed and the resulting data then propagated out to the outer boundary.

If such numerical studies were indeed successful they would open a new approach to the question of utmost physical importance: Does the resulting binary black hole initial data suppress junk radiation? The sole data needed on a single large surface in the asymptotic region surrounding the system distinguishes this approach from other solutions to the constraint problem which rely on elliptic equations. Whether this feature improves the physical content and control of the initial data is again a matter 
for numerical investigation.

\section{Acknowledgments}

IR and JW were supported in part by the NKFIH grant K-115434 and by NSF grant PHY-1505965 to the University of Pittsburgh, respectively.

\section{Appendix}

Here we give details of the spin-weight decomposition of some additional terms that are essential for the implementation of a numerical code.

\section{Terms involving the lapse $\widehat{N}$}

Using the notation $\widehat{\mathbb{N}}=\widehat{N}$, we obtain

$$
\begin{aligned}
& \widehat{D}^{l} \widehat{D}_{l} \widehat{N}=\widehat{\gamma}^{k l}\left[\widehat{D}_{k} \mathbb{D}_{l} \widehat{N}\right]=\widehat{\gamma}^{k l}\left[\mathbb{D}_{k} \mathbb{D}_{l} \widehat{N}-C^{f}{ }_{k l} \mathbb{D}_{f} \widehat{N}\right] \\
& =\mathbb{d}^{-1}\left\{\mathrm{a} q^{k l}-\frac{1}{2}\left[\mathrm{~b} \bar{q}^{k} \bar{q}^{l}+\overline{\mathrm{b}} q^{k} q^{l}\right]\right\}\left[\mathbb{D}_{k} \mathbb{D}_{l} \widehat{N}-\frac{1}{2} C_{k l}^{f}\left[q_{f} \bar{q}^{e}+\bar{q}_{f} q^{e}\right] \mathbb{D}_{e} \widehat{N}\right] \\
& =\frac{1}{2} \mathbb{d}^{-1}\left[a\{(ð \bar{\partial} \widehat{\mathbb{N}})-\mathbb{B}(\bar{\partial} \widehat{\mathbb{N}})\}-\varpi\left\{\left(\bar{\partial}^{2} \widehat{\mathbb{N}}\right)-\frac{1}{2} \overline{\mathbb{A}}(\bar{\partial} \widehat{\mathbb{N}})-\frac{1}{2} \overline{\mathbb{C}}(ð \widehat{\mathbb{N}})\right\}+" C C "\right] \text {. }
\end{aligned}
$$

By virtue of the relation $\dot{\widehat{n}}_{k}=\widehat{n}^{l} D_{l} \widehat{n}_{k}=-\widehat{D}_{k}(\ln \widehat{N})$ we also have

$$
q^{i} \dot{\hat{n}}_{i}=-\widehat{\mathbb{N}}^{-1} \precsim \widehat{\mathbb{N}}
$$

and

$$
\mathbb{k}^{i} \dot{\widehat{n}}_{i}=-(2 \mathbb{d} \widehat{\mathbb{N}})^{-1}\{(ð \widehat{\mathbb{N}})[\mathrm{a} \overline{\mathbb{k}}-\overline{\mathrm{b}} \mathbb{k}]+" C C "\}
$$

\section{Terms involving the shift $\widehat{N}^{i}$ and $\mathbf{K}_{l}^{l}$}

By making use of the relations

$$
\widetilde{\mathbb{N}}=q_{i} \widehat{N}^{i}=q_{i} \widehat{\gamma}^{i j} \widehat{N}_{j}=\mathbb{d}^{-1}\left(\mathrm{a} q^{j}-\mathfrak{b} \bar{q}^{j}\right) \widehat{N}_{j}=\mathbb{d}^{-1}(\mathrm{a} \mathbb{N}-\mathfrak{b} \overline{\mathbb{N}})
$$

or alternatively

$$
\mathbb{N}=q^{l} \widehat{N}_{l}=q^{l} \widehat{\gamma}_{l k} \widehat{N}^{k}=\left(\mathrm{a} q_{k}+\mathrm{b} \bar{q}_{k}\right) \widehat{N}^{k}=\mathrm{a} \widetilde{\mathbb{N}}+\mathrm{b} \overline{\widetilde{\mathbb{N}}},
$$

the Lie derivative $\mathscr{L}_{\widehat{n}}\left(\mathbf{K}_{l}^{l}\right)$ appearing in (2.15) can be expressed as

$$
\begin{aligned}
\mathscr{L}_{\widehat{n}}\left(\mathbf{K}_{l}^{l}\right) & =\widehat{n}^{i} D_{i} \mathbf{K}_{l}^{l}=\widehat{N}^{-1}\left[\left(\partial_{\rho}\right)^{i}-\widehat{N}^{i}\right] D_{i} \mathbf{K}_{l}^{l}=\widehat{N}^{-1}\left[\partial_{\rho} \mathbf{K}_{l}^{l}-\widehat{N}^{i} \mathbb{D}_{i} \mathbf{K}_{l}^{l}\right] \\
& =\mathscr{L}_{\widehat{n}} \mathbb{K}=\widehat{\mathbb{N}}^{-1}\left[\left(\partial_{\rho} \mathbb{K}\right)-\frac{1}{2} \widetilde{\mathbb{N}}(\bar{\partial} \mathbb{K})-\frac{1}{2} \overline{\mathbb{N}}(ð \mathbb{K})\right],
\end{aligned}
$$


where

$$
\mathbb{K}=\mathbf{K}_{l}^{l}=\widehat{\gamma}^{k l} \mathbf{K}_{k l}
$$

and we have used $\widehat{N}^{i} D_{i} \mathbf{K}_{l}^{l}=\widehat{N}^{i} \mathbb{D}_{i} \mathbf{K}_{l}^{l}=\frac{1}{2} \widehat{N}^{i}\left(q_{i} \bar{q}^{j}+\bar{q}_{i} q^{j}\right) \mathbb{D}_{j} \mathbf{K}_{l}^{l}$.

\section{Terms involving the trace-free part of $\mathbf{K}_{k l}$}

By setting

$$
\stackrel{\circ}{\mathbb{K}}_{q q}=q^{k} q^{l} \stackrel{\circ}{\mathbf{K}}_{k l}
$$

and

$$
\stackrel{\circ}{\mathbb{K}}_{q \bar{q}}=q^{k} \bar{q}^{l} \stackrel{\circ}{\mathbf{K}}_{k l}
$$

in virtue of (2.6), we obtain

$$
\stackrel{\circ}{\mathbf{K}}_{i j}=\frac{1}{2} q_{i j} \stackrel{\circ}{\mathbb{K}}_{q \bar{q}}+\frac{1}{4}\left[q_{i} q_{j} \overline{\stackrel{o}{\mathbb{K}}_{q q}}+\bar{q}_{i} \bar{q}_{j} \stackrel{\circ}{\mathbb{K}}_{q q}\right] .
$$

Note that, since $\stackrel{\circ}{\mathbf{K}}_{k l}$ is trace-free, $\stackrel{\circ}{\mathbb{K}}_{q \bar{q}}$ and $\stackrel{\circ}{\mathbb{K}}_{q q}$ are not functionally independent. Indeed, the trace-free condition $\widehat{\gamma}^{k l} \stackrel{\circ}{\mathbf{K}}_{k l}=0$ implies

$$
\stackrel{\circ}{\mathbb{K}}_{q \bar{q}}=(2 \mathrm{a})^{-1}\left[\mathrm{~b} \overline{\mathbb{K}_{q q}}+\overline{\mathbb{b}} \stackrel{\circ}{\mathbb{K}}_{q q}\right] .
$$

For both $\mathrm{a}^{-1}$ and $\stackrel{\circ}{\mathbb{K}}_{q \bar{q}}$, to be well-defined a cannot vanish. This is guaranteed because $\widehat{\gamma}_{i j}$ is a positive definite Riemannian metric so that $\mathbb{d}=\mathrm{a}^{2}-\mathbb{b} \overline{\mathrm{b}}$ must be positive.

We then have

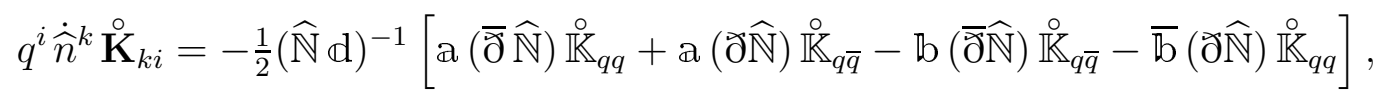

$$
\begin{aligned}
& q^{i} \widehat{D}^{k}{\stackrel{\circ}{\mathbf{K}_{k i}}}_{k}=\frac{1}{2} \mathbb{d}^{-1}\left\{\mathrm{a} \bar{\partial} \stackrel{\circ}{\mathbb{K}}_{q q}+\mathrm{a} \partial \stackrel{\circ}{\mathbb{K}}_{q \bar{q}}-\mathrm{b} \bar{\partial} \stackrel{\circ}{\mathbb{K}}_{q \bar{q}}-\overline{\mathrm{b}} \partial \stackrel{\circ}{\mathbb{K}}_{q q}\right\} \\
& -\frac{\mathrm{a}}{4 \mathbb{d}}\left\{3 \overline{\mathbb{B}} \stackrel{\circ}{\mathbb{K}}_{q q}+3 \mathbb{B} \stackrel{\circ}{\mathbb{K}}_{q \bar{q}}+\mathbb{A} \stackrel{\circ}{\mathbb{K}}_{q \bar{q}}+\mathbb{\mathbb { C }} \overline{\mathbb{K}_{q q}}\right\}
\end{aligned}
$$

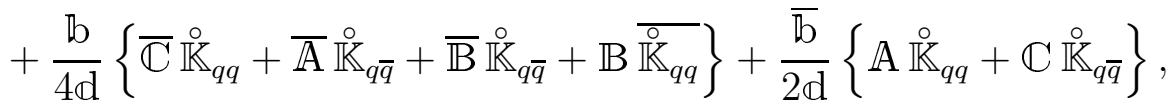

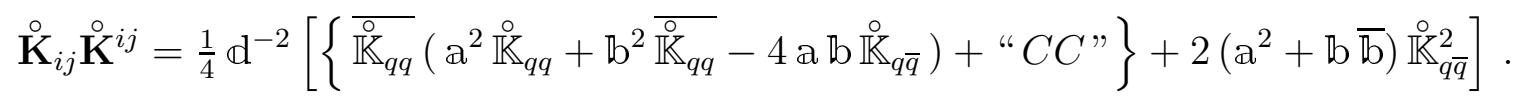




\section{The determination of $\mathscr{L}_{\widehat{n}} \mathbf{k}_{l}$ and $\mathscr{L}_{\rho} \mathbf{k}_{i}$}

The Lie derivative $\mathscr{L}_{\widehat{n}} \mathbf{k}_{l}$ appearing in (2.16), can be re-expressed as follows. First, note that

$$
\left(\mathscr{L}_{\widehat{n}} \mathbf{k}_{l}\right) \widehat{n}^{l}=\mathscr{L}_{\widehat{n}}\left(\mathbf{k}_{l} \widehat{n}^{l}\right)=0
$$

which implies

$$
\mathscr{L}_{\widehat{n}} \mathbf{k}_{l}=\widehat{\gamma}_{l}^{i} \mathscr{L}_{\widehat{n}} \mathbf{k}_{i}
$$

Then, it is straightforward to verify that

$$
\begin{aligned}
\mathscr{L}_{\widehat{n}} \mathbf{k}_{l} & =\widehat{\gamma}_{l}^{i} \mathscr{L}_{\widehat{n}} \mathbf{k}_{i}=\widehat{N}^{-1} \widehat{\gamma}_{l}^{i}\left[\mathscr{L}_{\rho} \mathbf{k}_{i}-\mathscr{L}_{\widehat{N}} \mathbf{k}_{i}\right] \\
& =\widehat{N}^{-1}\left[\widehat{\gamma}_{l}^{i}\left(\mathscr{L}_{\rho} \mathbf{k}_{i}\right)-\widehat{N}^{f} \widehat{D}_{f} \mathbf{k}_{l}-\mathbf{k}_{f} \widehat{D}_{l} \widehat{N}^{f}\right] \\
& =\widehat{N}^{-1}\left[\widehat{\gamma}_{l}^{i}\left(\mathscr{L}_{\rho} \mathbf{k}_{i}\right)-\widehat{N}^{f} \mathbb{D}_{f} \mathbf{k}_{l}-\mathbf{k}_{f} \mathbb{D}_{l} \widehat{N}^{f}\right]
\end{aligned}
$$

where in the second line we have used the torsion free property of the connection when evaluating $\mathscr{L}_{\widehat{N}} \mathbf{k}_{i}$.

In determining $q^{l} \mathscr{L}_{\widehat{n}} \mathbf{k}_{l}$ we use

$$
q^{l} \widehat{\gamma}_{l}^{i}\left(\mathscr{L}_{\rho} \mathbf{k}_{i}\right)=q^{l} q_{l}{ }^{i}\left(\mathscr{L}_{\rho} \mathbf{k}_{i}\right)=\left(\partial_{\rho} \mathbb{k}\right)
$$

and

$$
q^{l}\left[\widehat{N}^{f} \mathbb{D}_{f} \mathbf{k}_{l}+\mathbf{k}_{f} \mathbb{D}_{l} \widehat{N}^{f}\right]=\frac{1}{2}[\widetilde{\mathbb{N}} \bar{\partial} \mathbb{k}+\overline{\widetilde{\mathbb{N}}} \partial \mathbb{k}]+\frac{1}{2}[\mathbb{k} \partial \overline{\widetilde{\mathbb{N}}}+\overline{\mathbb{k}} \partial \widetilde{\mathbb{N}}]
$$

Then

$$
q^{l} \mathscr{L}_{\widehat{n}} \mathbf{k}_{l}=\widehat{\mathbb{N}}^{-1}\left(\partial_{\rho} \mathbb{k}-\frac{1}{2}[\widetilde{\mathbb{N}} \bar{\partial} \mathbb{k}+\overline{\widetilde{\mathbb{N}}} \partial \mathbb{k}+\mathbb{k} \partial \overline{\widetilde{\mathbb{N}}}+\overline{\mathbb{k}} \partial \widetilde{\mathbb{N}}]\right)
$$

\section{The decomposition of $\widehat{D}_{k} \widehat{N}_{l}$}

We also need to evaluate the auxiliary expressions $q^{k} q^{l}\left(\widehat{D}_{k} \widehat{N}_{l}\right)$ and $\bar{q}^{k} q^{l}\left(\widehat{D}_{k} \widehat{N}_{l}\right)$. To do so, first notice that

$$
\widehat{D}_{k} \widehat{N}_{l}=\mathbb{D}_{k} \widehat{N}_{l}-C_{k l}^{f} \widehat{N}_{f}
$$

from which

$$
\begin{aligned}
q^{k} q^{l}\left(\widehat{D}_{k} \widehat{N}_{l}\right) & =q^{k} q^{l}\left(\mathbb{D}_{k} \widehat{N}_{l}\right)-q^{k} q^{l} C^{f}{ }_{k l}\left[\frac{1}{2}\left(q_{f} \bar{q}^{e}+\bar{q}_{f} q^{e}\right)\right] \widehat{N}_{e} \\
& =\varnothing \mathbb{N}-\frac{1}{2} \mathbb{C} \overline{\mathbb{N}}-\frac{1}{2} \mathbb{A} \mathbb{N} \\
\bar{q}^{k} q^{l}\left(\widehat{D}_{k} \widehat{N}_{l}\right) & =\bar{q}^{k} q^{l}\left(\mathbb{D}_{k} \widehat{N}_{l}\right)-\bar{q}^{k} q^{l} C^{f}{ }_{k l}\left[\frac{1}{2}\left(q_{f} \bar{q}^{e}+\bar{q}_{f} q^{e}\right)\right] \widehat{N}_{e} \\
& =\bar{\varnothing} \mathbb{N}-\frac{1}{2} \mathbb{B} \overline{\mathbb{N}}-\frac{1}{2} \overline{\mathbb{B}} \mathbb{N} .
\end{aligned}
$$




\section{Terms involving $\widehat{K}_{i j}$}

Before determining $q^{l}\left[\widehat{\gamma}^{e f} \mathbf{k}_{e} \widehat{K}_{f l}\right]$, we need to evaluate the extrinsic curvature $\widehat{K}_{i j}$ of $\mathscr{S}_{\rho}$ as given by (2.7),

$$
\begin{aligned}
\widehat{K}_{i j} & =\frac{1}{2} \mathscr{L}_{\widehat{n}} \widehat{\gamma}_{i j}=\frac{1}{2} \widehat{N}^{-1}\left[\mathscr{L}_{\rho} \widehat{\gamma}_{i j}-\left(\widehat{D}_{i} \widehat{N}_{j}+\widehat{D}_{j} \widehat{N}_{i}\right)\right] \\
& =\frac{1}{2} \widehat{\mathbb{N}}^{-1}\left[\left(\partial_{\rho} \mathrm{a}\right) q_{i j}+\frac{1}{2}\left[\left(\partial_{\rho} \mathrm{b}\right) \bar{q}_{i} \bar{q}_{j}+\left(\partial_{\rho} \overline{\mathrm{b}}\right) q_{i} q_{j}\right]-\left(\widehat{D}_{i} \widehat{N}_{j}+\widehat{D}_{j} \widehat{N}_{i}\right)\right],
\end{aligned}
$$

where in the last step (4.1) was applied. As a result,

$$
\begin{gathered}
\widehat{\mathbb{K}}=\widehat{K}^{l}{ }_{l}=\widehat{\gamma}^{i j} \widehat{K}_{i j}=\mathbb{d}^{-1}\left\{\mathrm{a} q^{i j}-\frac{1}{2}\left[\mathrm{~b} \bar{q}^{i} \bar{q}^{j}+\overline{\mathrm{b}} q^{i} q^{j}\right]\right\} \widehat{K}_{i j}=\frac{1}{2}(\widehat{\mathbb{N}} \mathbb{d})^{-1} \times \\
\times\left[\mathrm{a}\left\{\left(\partial_{\rho} \mathrm{a}\right)-q^{i} \bar{q}^{j}\left[\widehat{D}_{i} \widehat{N}_{j}+\widehat{D}_{j} \widehat{N}_{i}\right]\right\}-\mathbb{b}\left\{\left(\partial_{\rho} \overline{\mathrm{b}}\right)-\bar{q}^{i} \bar{q}^{j}\left(\widehat{D}_{i} \widehat{N}_{j}\right)\right\}\right]+" C C " \\
=\frac{1}{2}(\widehat{\mathbb{N}} \mathbb{d})^{-1}\left\{\mathrm{a}\left[\left(\partial_{\rho} \mathrm{a}\right)-(\overline{\mathrm{d}} \mathbb{N})+\overline{\mathbb{B}} \mathbb{N}\right]\right. \\
\left.-\mathbb{b}\left[\left(\partial_{\rho} \overline{\mathrm{b}}\right)-(\overline{\mathrm{d}} \overline{\mathbb{N}})+\frac{1}{2} \overline{\mathbb{C}} \mathbb{N}+\frac{1}{2} \overline{\mathbb{A}} \overline{\mathbb{N}}\right]\right\}+" C C " .
\end{gathered}
$$

Now set

$$
\begin{array}{r}
\widehat{\mathbb{K}}_{q q}=q^{i} q^{j} \widehat{K}_{i j}=\frac{1}{2} \widehat{\mathbb{N}}^{-1}\left\{2 \partial_{\rho} \mathfrak{b}-2 \partial \mathbb{N}+\mathbb{C} \overline{\mathbb{N}}+\mathbb{A} \mathbb{N}\right\} \\
\widehat{\mathbb{K}}_{q \bar{q}}=q^{i} \bar{q}^{j} \widehat{K}_{i j}=\frac{1}{2} \widehat{\mathbb{N}}^{-1}\left\{2 \partial_{\rho \mathrm{a}}-\overline{\mathrm{\partial}} \mathbb{N}-\partial \overline{\mathbb{N}}+\mathbb{B} \overline{\mathbb{N}}+\overline{\mathbb{B}} \mathbb{N}\right\} .
\end{array}
$$

Then, because the symmetric 2-tensor $\widehat{K}_{l}^{l}$ is determined by three real functions, it follows that $\widehat{\mathbb{K}}_{q \bar{q}}, \widehat{\mathbb{K}}_{q q}$ and $\widehat{\mathbb{K}}$ are functionally dependent. In determining their algebraic relation we introduce the auxiliary variables

$$
\begin{aligned}
& \star \widehat{\mathbb{K}}_{q \bar{q}}=q^{i} \bar{q}^{j}\left[\widehat{K}_{i j}-\frac{1}{2} \widehat{\gamma}_{i j} \widehat{K}_{l}^{l}\right]=\widehat{\mathbb{K}}_{q \bar{q}}-\mathrm{a} \widehat{\mathbb{K}}, \\
& \star \widehat{\mathbb{K}}_{q q}=q^{i} q^{j}\left[\widehat{K}_{i j}-\frac{1}{2} \widehat{\gamma}_{i j} \widehat{K}_{l}^{l}\right]=\widehat{\mathbb{K}}_{q q}-\mathfrak{b} \widehat{\mathbb{K}} .
\end{aligned}
$$

The analog of the trace relation (A.11) then gives

$$
\star \widehat{\mathbb{K}}_{q \bar{q}}=(2 \mathrm{a})^{-1}\left[\bar{b} \overline{\widehat{\mathbb{K}}_{q q}}+\bar{b} \star \widehat{\mathbb{K}}_{q q}\right],
$$

from which it follows, in virtue of (A.28) and (A.29),

$$
\widehat{\mathbb{K}}_{q \bar{q}}=\mathrm{a}^{-1}\left\{\mathbb{d} \cdot \widehat{\mathbb{K}}+\frac{1}{2}\left[\mathfrak{b} \overline{\widehat{\mathbb{K}}_{q q}}+\overline{\mathrm{b}} \widehat{\mathbb{K}}_{q q}\right]\right\} .
$$

Then, by making use of all the prior variables related to $\widehat{K}^{i j}$, we obtain

$$
q_{i} q_{j} \widehat{K}^{i j}=\mathbb{d}^{-2}\left[\mathrm{a}^{2} \widehat{\mathbb{K}}_{q q}+\mathrm{b}^{2} \overline{\widehat{\mathbb{K}}_{q q}}-2 \mathrm{a} \mathfrak{b} \widehat{\mathbb{K}}_{q \bar{q}}\right]
$$

and

$$
q_{i} \bar{q}_{j} \widehat{K}^{i j}=\mathbb{d}^{-2}\left[\left(\mathrm{a}^{2}+\mathrm{b} \overline{\mathrm{b}}\right) \widehat{\mathbb{K}}_{q \bar{q}}-\mathrm{a} \overline{\mathrm{b}} \widehat{\mathbb{K}}_{q q}-\mathrm{a} \mathrm{b} \overline{\widehat{\mathbb{K}}_{q q}}\right]
$$


These relations, along with (A.10), imply

$$
\begin{aligned}
\stackrel{\circ}{\mathbf{K}}_{i j} \widehat{K}^{i j}=\frac{1}{4} \mathbb{d}^{-2}[ & \left.2 \stackrel{\circ}{\mathbb{K}}_{q \bar{q}}\left(\left[\mathrm{a}^{2}+\mathrm{b} \overline{\mathrm{b}}\right)\right] \widehat{\mathbb{K}}_{q \bar{q}}-\mathrm{a}\left[\overline{\mathrm{b}} \widehat{\mathbb{K}}_{q q}+\mathrm{b} \overline{\mathbb{K}}_{q q}\right]\right) \\
& \left.+\left\{\overline{\mathbb{K}_{q q}}\left[\mathrm{a}^{2} \widehat{\mathbb{K}}_{q q}+\mathrm{b}^{2} \overline{\mathbb{K}}_{q q}-2 \mathrm{a} \mathfrak{b} \widehat{\mathbb{K}}_{q \bar{q}}\right]+" C C "\right\}\right]
\end{aligned}
$$

and

$\widehat{K}_{i j} \widehat{K}^{i j}=\frac{1}{4} \mathbb{d}^{-2}\left\{\overline{\widehat{\mathbb{K}}_{q q}}\left[\mathrm{a}^{2} \widehat{\mathbb{K}}_{q q}+\mathrm{b}^{2} \widehat{\mathbb{K}}_{q q}-4 \mathrm{a} b \widehat{\mathbb{K}}_{q \bar{q}}\right]+" C C "\right\}+\frac{1}{2} \mathbb{d}^{-2}\left(\mathrm{a}^{2}+\mathrm{b} \overline{\mathrm{b}}\right) \widehat{\mathbb{K}}_{q \bar{q}}^{2}$.

Finally, the spin-weighted analogue of (A.6) is

$$
\mathscr{L}_{\widehat{n}}\left(\widehat{K}_{l}^{l}\right)=\mathscr{L}_{\widehat{n}} \widehat{\mathbb{K}}=\widehat{\mathbb{N}}^{-1}\left[\left(\partial_{\rho} \widehat{\mathbb{K}}\right)-\frac{1}{2} \widetilde{\mathbb{N}}(\bar{\oslash} \widehat{\mathbb{K}})-\frac{1}{2} \overline{\widetilde{N}}(ð \widehat{\mathbb{K}})\right] .
$$

\section{Terms involving starred quantities}

By virtue of (2.7) and (2.11),

$$
\stackrel{\star}{K}_{i j}=\widehat{N} \widehat{K}_{i j}
$$

Accordingly, as a consequence of (A.24)-(A.35),

$$
\begin{aligned}
\stackrel{\star}{\mathbb{K}}=\frac{1}{2} \mathbb{d}^{-1}\{\mathrm{a}[ & \left.\left(\partial_{\rho} \mathrm{a}\right)-(\overline{\mathrm{\partial}} \mathbb{N})+\overline{\mathbb{B}} \mathbb{N}\right] \\
& \left.-\mathrm{b}\left[\left(\partial_{\rho} \overline{\mathrm{b}}\right)-(\overline{\mathrm{d}} \overline{\mathbb{N}})+\frac{1}{2} \overline{\mathbb{C}} \mathbb{N}+\frac{1}{2} \overline{\mathbb{A}} \overline{\mathbb{N}}\right]\right\}+" C C " .
\end{aligned}
$$

By now setting

$$
\begin{gathered}
{\stackrel{\star}{\mathbb{K}_{q q}}}_{q}=q^{i} q^{j} \stackrel{\star}{K}_{i j}=\frac{1}{2}\left\{2 \partial_{\rho} \mathrm{b}-2 ð \mathbb{N}+\mathbb{C} \overline{\mathbb{N}}+\mathbb{A} \mathbb{N}\right\}, \\
\stackrel{\star}{\mathbb{K}}_{q \bar{q}}=q^{i} \bar{q}^{j} \stackrel{\star}{K}_{i j}=\frac{1}{2}\left\{2 \partial_{\rho} \mathrm{a}-\bar{\partial} \mathbb{N}-ð \overline{\mathbb{N}}+\mathbb{B} \overline{\mathbb{N}}+\overline{\mathbb{B}} \mathbb{N}\right\}
\end{gathered}
$$

we obtain

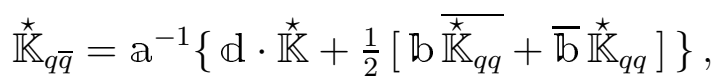

while (5.10) and (5.11) follow straightforwardly from (A.34) and (A.35).

\section{References}

[1] Lichnerowicz A (1944) L'integration des Equations de la Gravitation Relativiste et le Probleme des $n$ Corps, J. Math. Pures Appl., 23, 39-63

[2] York J W (1972) Role of conformal three-geometry in the dynamics of gravitation, Phys. Rev. Letters 28, 1082-1085 
[3] York J W (1974) Covariant decompositions of symmetric tensors in the theory of gravitation, Ann. Inst. Henri Poincaré A 21 319-332

[4] Cook G B (2000) Initial data for numerical relativity, Living Rev. Relativity 35

[5] Gourghoulon E (2007) Construction of initial data for 3+1 numerical relativity, J. Phys. Conf. Ser. 91, 012001

[6] Rácz I (2016) Constrains as evolutionary systems, Class. Quant. Grav. 33015014

[7] Rácz I (2014) Is the Bianchi identity always hyperbolic?, Class. Quant. Grav. 31 155004

[8] Rácz I (2015) Cauchy problem as a two-surface based 'geometrodynamics', Class. Quant. Grav. 32015006

[9] Rácz I (2014) Dynamical determination of the gravitational degrees of freedom, arXiv:1412.0667

[10] Rácz I and Winicour J (2015) Black hole initial data without elliptic equations, Phys. Rev. D 91, 124013

[11] Chu T (2014) Including realistic tidal deformations in binary black-hole initial data, Phys. Rev. D 89064062

[12] Lovelace G (2009) Reducing spurious gravitational radiation in binary-blackhole simulations by using conformally curved initial data, Class.Quant.Grav. 26 114002

[13] Matzner R A, Huq M F and Shoemaker D (1999) Initial data and coordinates for multiple black hole systems, Phys. Rev. D 59024015

[14] Bonning E, Marronetti P, Neilson D and Matzner R A (2003) Physics and initial data for multiple black hole spacetimes, Phys. Rev. D. 68044019

[15] Rácz I (2016) On the ADM charges of multiple black holes, arXiv:1608.02283

[16] Babiuc Hamilton M (2018) Hyperbolic initial data for nontrivial spacetimes, Presentation at the April 2018 APS Meeting, https: / /absuploads.aps.org/presentation.cfm?pid=14323

[17] Nakonieczna A, Nakonieczny L and Rácz I (2017) Black hole initial data by numerical integration of the parabolic-hyperbolic form of the constraints, arXiv:1712.00607 
[18] Rácz I and Winicour J (2016) On solving the constraints by integrating a strongly hyperbolic system, arXiv:1601.05386 [gr-qc]

[19] Beyer F, Escobar L and Frauendiener J (2017) Asymptotics of solutions of a hyperbolic formulation of the constraint equations, Class. Quant. Grav. 34205014

[20] Maliborski M (2017) private communication

[21] Schell C (2017) Spectral approach to the axisymmetric evolution of Einstein's vacuum equations, PhD Dissertation, Freien Universität Berlin, www.diss.fuberlin.dediss/receiveFUDISS_thesis_000000106393

[22] Choquet-Bruhat Y (2009) General relativity and Einstein's equations, Oxford University Press Inc., New York

[23] Wald R M (1984) General relativity, University of Chicago Press

[24] Newman E T and Penrose R (1966) Note on the Bondi-Metzner-Sachs Group, J. Math. Phys. 7, 863-870

[25] Goldberg J N, Macfarlane A J, Newman E T, Rohrlich F and Sudarshan E C G (1967) Spin-s Spherical Harmonics and ð, J. Math. Phys. 8, 2155-2161

[26] Gómez R, Lehner L, Papadopoulos P and Winicour J (1997) The eth formalism in numerical relativity, Class. Quantum Grav. 14, 977-990

[27] Zlochower Y, Gómez R, Husa S, Lehner L and Winicour J (2003) Mode coupling in the nonlinear response of black holes, Phys. Rev. D 68, 084014

[28] LeFloch P G and Rácz I (2017), work in progress

[29] Beyer F, Daszuta B, Frauendiener J and Whale B (2014) Numerical evolutions of fields on the 2-sphere using a spectral method based on spin- or conformalweighted spherical harmonics, Class. Quant. Grav. 31075019

[30] Rácz I (2016) A simple method of constructing binary black hole initial data, arXiv:1605.01669

[31] Winicour J (2017) The algebraic-hyperbolic approach to the linearized gravitational constraints on a Minkowski background, Class. Quantum Grav. 34, 157001 